\title{
Failure of rewarding and locomotor stimulant doses of morphine to promote adult rat $50-\mathrm{kHz}$ ultrasonic vocalizations
}

\author{
Jennifer M. Wright • Lan Deng • Paul B. S. Clarke
}

Received: 14 February 2012 / Accepted: 9 June 2012 /Published online: 3 July 2012

(C) Springer-Verlag 2012

\begin{abstract}
Rationale Frequency-modulated $50-\mathrm{kHz}$ ultrasonic vocalizations (USVs) are emitted by adult rats in response to psychostimulants and non-pharmacological appetitive stimuli and thus have been proposed to model positive affect. Objective The main aim was to determine whether rewarding doses of morphine increase $50-\mathrm{kHz}$ call rate or alter the relative prevalence of the trill call subtype.

Methods In experiment 1, USVs were recorded from adult male Long-Evans rats after subchronic morphine $(1 \mathrm{mg} / \mathrm{kg}$ subcutaneous (SC)) administration, acute challenge with morphine ( 1 and $3 \mathrm{mg} / \mathrm{kg} \mathrm{SC}$ ) or amphetamine $(1 \mathrm{mg} / \mathrm{kg}$ IP, positive control), and in conjunction with locomotor activity tests with morphine ( 1 and $3 \mathrm{mg} / \mathrm{kg} \mathrm{SC}$ ). In experiments 2 and 3, the USV altering, rewarding, and locomotor effects of morphine were examined using a conditioned place preference (CPP) procedure.

Results In experiment 1 , morphine $(1 \mathrm{mg} / \mathrm{kg})$ initially suppressed calling; rats became tolerant to this effect with repeated exposure. Tested subsequently in singly- and pairtested rats, morphine markedly decreased USVs but significantly increased locomotor activity. In experiments 2 and 3, morphine produced a significant CPP without increasing either unconditioned or conditioned USV emission. Morphine did not detectably alter the relative prevalence of 50$\mathrm{kHz}$ call subtypes.
\end{abstract}

Electronic supplementary material The online version of this article (doi:10.1007/s00213-012-2776-z) contains supplementary material, which is available to authorized users.

J. M. Wright $\cdot$ L. Deng $\cdot$ P. B. S. Clarke $(\triangle)$

Department of Pharmacology and Therapeutics, McGill

University,

McIntyre Medical Building Rm. 1320,

3655 Promenade Sir William Osler,

Montreal, QC H3G 1Y6, Canada

e-mail: paul.clarke@mcgill.ca
Conclusions Although 50-kHz calls, and the trill call subtype in particular, have been proposed as an animal model of positive mood, not all euphoriant drugs acutely increase the rate of $50-\mathrm{kHz}$ calling or consistently promote trill calls.

Keywords Ultrasonic vocalizations · Rat · Morphine · Opioid · Amphetamine $\cdot$ Reward $\cdot$ Conditioned place preference $\cdot$ Locomotor activity

\section{Introduction}

Adult laboratory rats emit two broad categories of vocalizations in the ultrasonic range, commonly designated as "50kHz" and "22-kHz" calls (Brudzynski 2009; Wohr and Schwarting 2010). The 50-kHz call category encompasses a broad frequency range (30-90 kHz) (Kaltwasser 1990; Sales and Pye 1974) and comprises multiple subtypes, including flat (i.e., constant frequency) and at least 12 types of frequencymodulated (FM) calls (Wright et al. 2010). Recently, we have shown that the acoustic profile (i.e., relative prevalence of different call subtypes) can be modulated by drugs or social context (Wright et al. 2010, 2012), adding to the existing evidence that distinct information may be contained within the repertoire of 50-kHz USVs (e.g., Burgdorf et al. 2008; Simola et al. 2009; Wohr et al. 2008).

Whereas the 22-kHz calls appear to express distress or alarm (Covington and Miczek 2003; Litvin et al. 2007), 50-kHz calls are elicited by a number of appetitive stimuli (Burgdorf et al. 2010; Knutson et al. 2002). Consequently, adult rat 50-kHz calls (and FM calls in particular) have been proposed to be a measure of hedonia (Burgdorf and Moskal 2009) and have been described as a reliable indicator (Brudzynski 2007) and validated model (Burgdorf et al. 2010) of positive affective states. Consistent with this notion, the psychomotor stimulants amphetamine (AMPH) and cocaine both increase the $50-\mathrm{kHz}$ 
call rate and promote FM calls after systemic injection (e.g., Ahrens et al. 2009; Williams and Undieh 2010; Wright et al. 2010, 2012). Among the FM calls, it is the trill calls in particular that appear to be preferentially increased by these drugs (Wright et al. 2010, 2012). Psychostimulant drugs, however, are not only euphorigenic (Foltin and Fischman 1991) but are also anxiogenic in rodents and humans (Biala and Kruk 2007; File and Hyde 1979; Pellow et al. 1985); in high doses, these drugs can even produce acute psychosis (Angrist et al. 1974; Robinson and Becker 1986). Hence, it is important to note that trill calls are also affected by appetitive non-pharmacological manipulations. In particular, trill calls are also enhanced by testing rats in pairs (Wright et al. 2010), which itself is reported to be rewarding (Calcagnetti and Schechter 1992), whereas they appear less prevalent upon social separation (Wohr et al. 2008). Hence, on present evidence, the trill call subtype appears most closely associated with positive stimuli (Wright et al. 2010).

Opiate agonists form a second major class of euphorigenic drugs (Jasinski and Preston 1986; Zacny et al. 1994), but it is unclear whether they affect $50-\mathrm{kHz}$ USVs in a similar way to psychostimulants. Systemic morphine administration was initially reported to inhibit $50-\mathrm{kHz}$ calling (Haney and Miczek 1994, 1995; Vivian and Miczek 1993); however, these experiments were conducted in an aversive context (social stress) in which the vocalizations were probably acoustically distinct from those proposed to reflect positive affect (Wohr et al. 2008). Acute systemic morphine has also been combined with experimenterapplied tickling-like tactile stimulation, with no apparent drug effect on call rate (Panksepp and Burgdorf 2000). Acute morphine also failed to affect $50-\mathrm{kHz}$ calling rate in a novel testing environment; however, call rates were uniformly very low (i.e., $\leq 1$ call per minute) even in salinechallenged rats (Wohr and Schwarting 2009), suggesting that the environment itself may have generally inhibited USV production. In contrast, morphine reportedly induced $50-\mathrm{kHz}$ USVs in a reward context, i.e., conditioned place preference (CPP) (Burgdorf et al. 2001; Knutson et al. 1999). The latter studies, however, demonstrated only morphine-conditioned USVs and, importantly, the unconditioned effects of morphine (i.e., occurring during the CPP acquisition phase) were not reported.

In the absence of other behavioral manipulations, acute morphine administration tended to inhibit $50-\mathrm{kHz}$ call rates according to two recent studies (Hamed et al. 2012; Simola et al. 2012). These apparent (but statistically nonsignificant) suppressant effects occurred at relatively high doses of morphine of 5 or $10 \mathrm{mg} / \mathrm{kg}$ which may have nonspecifically inhibited motor output (Fernandes et al. 1977; Fog 1970); lower doses appeared ineffective (Simola et al. 2012). In the latter study, a preliminary analysis of call subtypes revealed no drug effect on trill calls (Simola et al. 2012). These two
USV studies suggest a possible dissociation between morphine's rewarding effects and $\mathrm{FM} 50-\mathrm{kHz}$ calls (and trill calls in particular); this inference is necessarily indirect, however, since rewarding drug effects were not measured.

Since FM $50-\mathrm{kHz}$ calls (and trill calls in particular) have been proposed to reflect positive affect in rats (see above), we hypothesized that after systemic administration morphine would promote trill calls and possibly increase the overall rate of $50-\mathrm{kHz}$ FM calls. This question was addressed in three experiments, using doses of morphine that are reportedly rewarding in CPP tests (Bardo et al. 1995). In experiment 1 , we initially tested individual rats with repeated morphine challenge; a low dose ( $1 \mathrm{mg} / \mathrm{kg} \mathrm{SC})$ was chosen to avoid catalepsy, which tends to occur above $5 \mathrm{mg} / \mathrm{kg}$ in drug-naïve rats (Fernandes et al. 1977; Fog 1970). We then tested the USV response to different doses of morphine (1 and $3 \mathrm{mg} / \mathrm{kg}$ ) and also to AMPH (1 mg/kg). Here, rats were tested both individually and with a cage mate. The latter condition served as a test of generalizability, since rats call more frequently and with a distinct acoustic profile in the presence of conspecifics (Brudzynski and Pniak 2002; Wright et al. 2010). Having noted a USVdepressant effect of morphine, we next studied ultrasonic calling in parallel with locomotor activity in order to test for sedation. Next, in experiment 2, the rewarding and locomotor effects of morphine were examined using a CPP procedure; here, USVs were recorded during the acquisition (conditioning) phase and during subsequent drug-free sessions where rats were restricted to either the saline- or morphine-paired floor texture. Finally, in experiment 3, USV recording was performed during the acquisition phase and during the free-choice CPP test session.

\section{Methods}

\section{Subjects}

Subjects were 64 experimentally-naïve male Long-Evans rats (Charles River Laboratories, St. Constant, Quebec, Canada) weighing $339 \pm 14 \mathrm{~g}$ (mean $\pm \mathrm{SD}$ ) (i.e., aged approximately 9 weeks) at the start of the experiment. Subjects were housed two per cage $\left(25 \times 48 \times 20 \mathrm{~cm}^{3}\right)$ in a temperature- and humidity-controlled colony room $\left(20-22{ }^{\circ} \mathrm{C}, 50-60 \%\right)$ at the McGill University Animal Resources Centre. The rats were maintained on a reverse 12:12 light/dark cycle, with lights off at 0700 hours. All behavioral testing took place during the dark phase of the cycle. Food and water were available ad libitum, except during testing. Before the start of the experiment, each rat was handled by the experimenter for approximately $3 \mathrm{~min}$ per day for 1-2 days (experiments 1 and 2) or 5 min per day over 6 days (experiment 3). All procedures were approved by the McGill Animal Care 
Committee in accordance with the guidelines of the Canadian Council on Animal Care.

\section{Locomotor activity and CPP apparatus}

Locomotor activity and CPP were both tested in rectangular, open-topped CPP cages $(58-\mathrm{cm}$ long $\times 29-\mathrm{cm}$ wide $\times 53-\mathrm{cm}$ high). Two floor textures were used as conditional stimuli: a mesh grid ( $1 \mathrm{~cm}^{2}$ stainless steel wire mesh) and a metal panel containing small holes $(4.8 \mathrm{~mm}$ diameter, set $6.4 \mathrm{~mm}$ apart). Rats do not show spontaneous preference for either floor texture (T. Scardochio and P. B. S. Clarke, unpublished observation). Square $(29 \times 29 \mathrm{~cm})$ tiles made of either flooring were mounted on melamine frames; two tiles completely covered the bottom of each CPP cage and, for experiment 2, one tile fit into each USV recording chamber. A video tracking system (EthoVision v 3.0, Noldus Information Technology, Leesburg, VA, USA) measured locomotor activity (expressed as the total horizontal distance moved) and the time spent on each floor texture (on CPP test day). To minimize visual cues, conditioning and testing in the CPP cages were conducted under darkroom lighting using a Kodak GBX-2 safelight filter (Vistek, Toronto, Ontario, Canada) which provided far-red (wavelength $>650 \mathrm{~nm}$ ) illumination.

Acoustic data acquisition

USV recordings in experiments 1 and 2 took place as previously described in clear Plexiglas experimental chambers (ENV-007CT, Med Associates, St Albans, VT). Please refer to Wright et al. (2012) for details concerning the USV recording apparatus and setup. All lights were off when rats were in the USV test chambers. For experiment 3, two ultrasound microphones were secured inside each CPP chamber at opposite corners, approximately $10 \mathrm{~cm}$ from the top (i.e., $40 \mathrm{~cm}$ above the floor). Sound-attenuating acoustic foam (Primacoustic, Port Coquitlam, British Columbia) enveloped the outside of the CPP chambers and extended $20 \mathrm{~cm}$ above the top of each chamber.

\section{Drugs}

Drugs were morphine sulfate pentahydrate (Sandoz, Boucherville, Quebec) and D-amphetamine sulfate (SigmaAldrich, Poole, UK). Morphine (1 and $3 \mathrm{mg} / \mathrm{kg}$, dose expressed as free base) was administered by subcutaneous (SC) injection into the flank. AMPH (1 mg/kg, dose as salt) was administered through intraperitoneal (IP) injection. Both drugs were dissolved in sterile $0.9 \%$ saline and administered in a volume of $1 \mathrm{ml} / \mathrm{kg}$.
Experimental protocol

\section{Experiment 1}

This experiment comprised three consecutive parts $(1.1-$ 1.3). The same 24 rats were used throughout. Rats were left undisturbed in their home cages during the 3 days between parts 1.1 and 1.2 , and the 8 days between parts 1.2 and 1.3.

Part 1.1: effects of repeated morphine administration on 50$\mathrm{kHz}$ ultrasonic vocalizations Repeated morphine exposure can produce sensitization to its rewarding effects (Lett 1989), and we anticipated that an analogous effect might occur with ultrasonic calling. Accordingly, the 24 subjects were randomly allocated to receive either morphine $(1 \mathrm{mg} /$ $\mathrm{kg} \mathrm{SC}, n=12)$ or saline $(n=12)$ daily over 3 days in the home cage prior to USV testing. On each of the following six test days, rats received an injection of morphine $(1 \mathrm{mg} / \mathrm{kg} \mathrm{SC})$ or saline (presented in alternating sequence) and were placed in a recording test chamber from 30 to $60 \mathrm{~min}$ post-injection. The order of testing was counterbalanced such that within each group of 12 rats, six rats on each test day received morphine and six rats received saline.

Part 1.2: effects of morphine and amphetamine on $50-\mathrm{kHz}$ ultrasonic vocalizations in rats tested individually or in pairs Part 1.2 occurred on eight consecutive days according to a fully parametric $2 \times 4$ within-subjects design, whereby each rat was tested once singly and once paired with a cage mate, under each of four drug conditions: morphine $(0,1$ and $3 \mathrm{mg} /$ $\mathrm{kg}, \mathrm{SC})$ or AMPH $(1 \mathrm{mg} / \mathrm{kg}, \mathrm{IP})$. The rats $(n=24)$ were placed in the recording chambers $30-60$ min post-injection. Cage mates were always tested under the same drug condition. The order of testing was counterbalanced as far as possible using a Williams square design.

Part 1.3: effect of morphine on $50-\mathrm{kHz}$ ultrasonic vocalizations and locomotor activity The same 24 rats were initially habituated to the locomotor test boxes for $10 \mathrm{~min}$. On the following 3 days, each rat was tested once with saline and morphine 1 and $3 \mathrm{mg} / \mathrm{kg}$ (SC) (order of testing was counterbalanced). Starting $20 \mathrm{~min}$ post-injection, half of the rats received a 20-min locomotor activity test session followed immediately by a 20 -min USV recording session. The remaining rats received the same two tests in the reverse order.

Experiment 2: morphine-conditioned place preference and $50-\mathrm{kHz}$ ultrasonic vocalizations

Subjects $(n=24)$ were not preexposed to morphine in the home cage since this had had no detectable effect on the USV responses in experiment 1 . Behavioral testing consisted 
of four main phases, extending over 12 consecutive days in total: habituation (day 1), conditioning (days 2-9), CPP test (day 10), and conditioned USV tests (days 11-12). On the first day (day 1), the 24 rats, which were all drug- and experimentally naïve, were habituated to the CPP chambers for $20 \mathrm{~min}$ on a layer of wood-chip bedding. Rats then underwent eight once-daily conditioning trials (days 2-9), whereby morphine ( 1 or $3 \mathrm{mg} / \mathrm{kg}$, depending on group, $n=12$ ) and saline were repeatedly paired (on alternating days) with a distinct floor texture which served as a tactile cue. Immediately following injection, half of the rats $(n=12)$ were conditioned in the CPP cages for $20 \mathrm{~min}$ and then promptly transferred to the USV recording chambers where they received an additional $20 \mathrm{~min}$ of contact with the same drug- or saline-paired floor texture; this order of conditioning was reversed for the other rats $(n=$ 12). On the CPP test day (day 10), rats were placed drug-free in the middle of the CPP cage, straddling the two floor tiles, and for the next $20 \mathrm{~min}$ were free to choose between the two floor textures. On the conditioned USV test days (days 11-12), each rat was recorded in the USV test chamber while being exposed for $20 \mathrm{~min}$ to the saline- or morphine-paired floor texture. This entire experiment was counterbalanced as far as possible in all respects, i.e., drug/floor-texture pairing, the order of drug vs. saline administration, position of morphine-paired floor texture within the test cage and order of floor texture presentation during the two USV test days. This experiment concluded 1 week later with two additional USV test sessions which served to demonstrate that the rats were capable of emitting high rates of calling in response to a drug. Here, the rats were tested for $20 \mathrm{~min}$ immediately after AMPH (1 mg/kg, IP) or saline, given in counterbalanced order.

\section{Experiment 3: 50-kHz ultrasonic vocalizations during acquisition and expression of morphine-conditioned place preference}

Experiment 3 differed from experiment 2 in the following respects. (1) All behavioral measures (i.e., USVs, locomotor activity, and place preference) were collected simultaneously in the same (CPP) apparatus. (2) In order to minimize possible experimenter-induced stress or anxiety, rats were handled more (see "Subjects" above) and underwent two (vs. one) habituation sessions to the CPP apparatus before the conditioning phase. (3) All rats $(n=16)$ were conditioned with only the lower morphine dose (i.e., $1 \mathrm{mg} / \mathrm{kg}$ ). (4) Testing for conditioned USVs was conducted during a single CPP test session during which the rats were free to choose between the drug- and saline-paired floor textures.

Analysis and classification of ultrasonic vocalizations

Acoustical analysis was performed as previously described (Wright et al. 2012) using Avisoft SASLab Pro (version 5.1,
Avisoft Bioacoustics). Calls were selected manually from spectrograms by an individual who was masked to the treatment condition. Each identified $50-\mathrm{kHz}$ call was classified into 1 of 14 distinct categories: complex, upward ramp, downward ramp, flat, short, split, step up, step down, multi step, trill, flat-trill combination, trill with jumps, inverted $U$, or composite (see Wright et al. 2010 for criteria for call identification and classification, several examples of each call type, as well as descriptive statistics relating to acoustic parameters). This method of manual call selection has been validated by surgical devocalization, and our method of classification is associated with high inter- and intra-rater reliability (Wright et al. 2010). Twenty-two kilohertz calls were rarely observed in the present study; only a small minority of rats in experiment 1.2 (mostly in the pairtested condition) emitted any $22-\mathrm{kHz}$ USVs. Therefore these calls were not analyzed further.

Data analysis and statistics

Data were analyzed using commercial software (Systat v11, SPSS, Chicago, IL; GraphPad Prism 4, GraphPad Software, La Jolla, CA). In experiments 1.1 and 1.2, USVs that occurred during min 1, 6, 11, 16, 21, and 26 of the 30-min session were counted and classified. For experiment 1.3, USV analysis was performed for min 1, 11, and 20 of the 20 -min session. In experiment 1.1 , one rat was a serious outlier (i.e., $>3$ standard deviations from the mean) rendering the use of ANOVA invalid and was therefore excluded from the statistical analysis. In experiment 1.2, rats were tested both singly and with their cage mate. When rats were tested in pairs, the call rate was divided by two. Therefore, there were $n=12$ pairs for part 2 and the data are expressed as calls per minute per rat. One rat pair was a serious outlier and was therefore excluded from analysis. For experiments 2 and 3, USV analysis was performed for min 3, 8, 13, and 18 of the 20 -min session and 3, 8, 13, 18, 23, 28, 33, and 38 of the 40-min session, respectively. However, all USVs that occurred during the entire 20-min CPP test were counted and classified. Due to a technical problem, locomotor data collection failed for four out of the 24 rats during the third conditioning trial in experiment 2 . Therefore, these rats were excluded from the locomotor activity analysis for that trial. Repeated measures ANOVA or Friedman's test was performed, where appropriate, and pairwise comparisons were performed using paired $t$ tests or Wilcoxon tests. The choice of test depended on the distribution of the raw data. ANOVA determined the effect of test pair (i.e., morphine minus saline difference scores for USV rate for each of the three morphine/saline tests in experiment 1.1), group (i.e., home cage morphine preexposure), and drug (i.e., morphine dose in experiment 1.3). ANOVA $\mathrm{p}$ values were subject to the Huynh-Feldt correction, where appropriate, and multiple 
comparisons were subject to Holm-Bonferroni correction. For all analyses, a two-tailed $p$ value $<5 \%$ (after any correction) was considered significant.

\section{Results}

Experiment 1

Part 1.1 Acute effects of morphine on $50-\mathrm{kHz}$ calling are shown in Fig. 1. Morphine preexposure (i.e., $3 \times 1 \mathrm{mg} / \mathrm{kg} \mathrm{SC}$ in the home cage) failed to alter the acute effects of the drug, and therefore rats from both groups were pooled for all subsequent analyses. Morphine significantly inhibited calling, but only in the first pair of morphine/saline tests (Wilcoxon $Z=$ $3.05, p<0.01$; Fig. 1). Tolerance developed by the third test pair as evidenced by a significant difference in the MOR-SAL difference score between the first and third test pairs (test pair, $\left.F_{2,42}=5.31, p=0.02 ; t_{22}=2.50, p<0.05\right)$.

Part 1.2 Rats tended to emit more calls (on a per rat basis) when tested with their cage mate than when tested alone. This difference was statistically significant in the saline, morphine $1 \mathrm{mg} / \mathrm{kg}$, and AMPH conditions (Wilcoxon $Z$ scores, 2.762.93, $p<0.02$ ); Fig. 2). Morphine ( 3 but not $1 \mathrm{mg} / \mathrm{kg}$ ) significantly decreased the call rate when rats were tested singly or in pairs (Wilcoxon $Z=2.31$ and 2.80, respectively, $p<0.05$; Fig. 2). AMPH increased the call rate, as expected, under both conditions (Wilcoxon $Z=2.93$ and $p<0.01$; Fig. 2). The timecourse of USV emission during the test sessions is shown in Fig. 3.

Part 1.3 USVs were tested either before or after locomotor activity, depending on the group ( $n=12$ rats per group). Data from these two groups were pooled, since neither the locomotor activity nor the USV rate revealed a significant

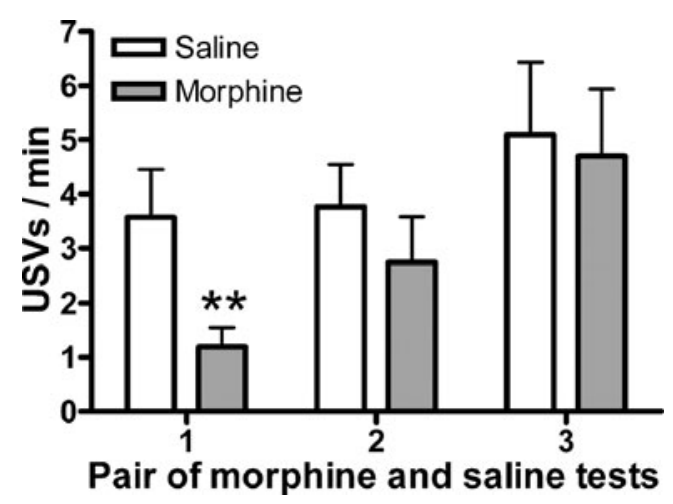

Fig. 1 Experiment 1.1: morphine $(1 \mathrm{mg} / \mathrm{kg}, \mathrm{SC})$ initially suppressed $50-\mathrm{kHz}$ calling, but rats became tolerant to this effect with repeated testing. The $y$-axis represents mean $+\mathrm{SEM} 50-\mathrm{kHz}$ USVs per minute under saline (open bars) and $1 \mathrm{mg} / \mathrm{kg}$ morphine (gray bars). ${ }^{* *} p<$ $0.01(n=24)$

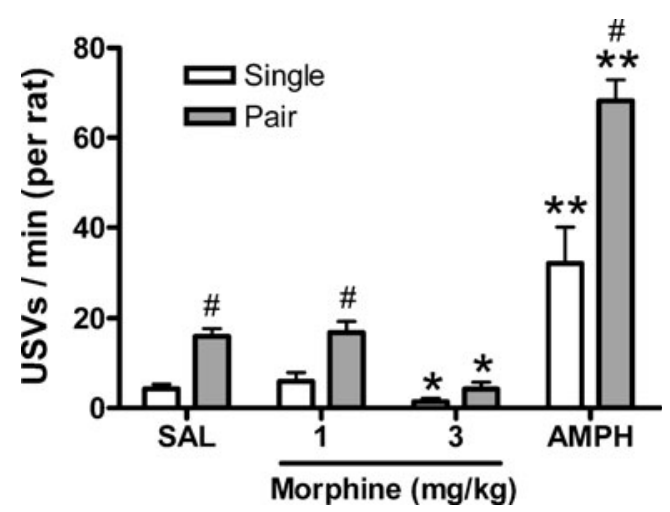

Fig. 2 Experiment 1.2: morphine dose-dependently inhibited calling in both singly- (open bars) and pair-tested (gray bars) rats. The $y$-axis represents mean+SEM USVs per minute (on a per rat basis). Each rat was tested under all eight conditions ( $n=11$ rat pairs). ${ }^{*} p<0.05,{ }^{* *} p<$ 0.01 vs. respective saline condition; $\# p<0.02$ vs. tested singly under the same drug treatment

between-group difference. Both doses of morphine (1 and $3 \mathrm{mg} / \mathrm{kg}$ ) increased total locomotor activity per 20-min session (drug: $F_{2,44}=7.68, p<0.01 ; 1 \mathrm{mg} / \mathrm{kg}$ vs. saline, $t_{23}=4.5, p<$ $0.001 ; 3 \mathrm{mg} / \mathrm{kg}$ vs. saline, $t_{23}=3.0, p<0.01$; Fig. 4 a) but decreased the call rate (Friedman $Q_{2}=25.97, p<0.0001$; Wilcoxon tests: saline vs. $1 \mathrm{mg} / \mathrm{kg}, Z=3.67, p<0.001$; saline vs. $3 \mathrm{mg} / \mathrm{kg}, Z=3.81, p<0.001$; Fig. 4b). The higher dose of morphine virtually abolished calling (Fig. 4b).

\section{Experiment 2}

During the CPP conditioning phase, the call rate and locomotor activity did not differ significantly between rats tested 0-20 or 20-40 min after injection. Therefore, rats were pooled for the remainder of the analysis. During the conditioning phase, morphine (especially at $3 \mathrm{mg} / \mathrm{kg}$ ) tended to reduce the USV emission rate, but this apparent effect was nonsignificant (Fig. 5a, c). Morphine also failed to affect locomotor activity, except for a modest, but nonsignificant, stimulant effect that emerged at the $3-\mathrm{mg} / \mathrm{kg}$ dose during later conditioning sessions (paired $t$ test between saline and $3 \mathrm{mg} / \mathrm{kg}$ morphine for trials 3 and 4, with Holm-Bonferroni corrections: $t_{9}=3.09$ and $t_{11}=2.73, p=0.052$ and 0.059 , respectively; Fig. 5b, d). On the CPP test day, both doses of morphine produced a highly significant place preference (one sample $t$ tests for MOR-SAL difference scores: $1 \mathrm{mg} /$ $\mathrm{kg}, t_{11}=5.94, p<0.001 ; 3 \mathrm{mg} / \mathrm{kg}, t_{11}=2.71, p<0.05, n=12$ rats per dose; Fig. 6a).

There was no significant difference in USV emission when rats were confined drug-free to the morphine-paired vs. salinepaired floor textures (Fig. 6b). The final tests with AMPH and saline confirmed the well-established unconditioned increase in call rate in response to this drug (Wilcoxon $Z=4.11, p<$ $0.0001, n=24$; Fig. $6 c$ ). 
Fig. 3 Experiment 1.2: time course of USV emission following saline (open circles), morphine ( 1 or $3 \mathrm{mg} / \mathrm{kg}$, SC; downward/solid and upward/ open triangles, respectively) or AMPH (1 mg/kg, IP; solid circle) when rats were singly tested (a) and pair-tested (b). The $y$-axes represent mean + SEM USVs per minute (on a per rat basis) a.

Singly-tested

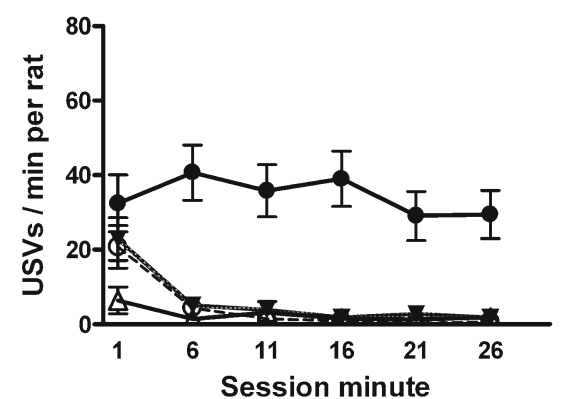

b. Pair-tested

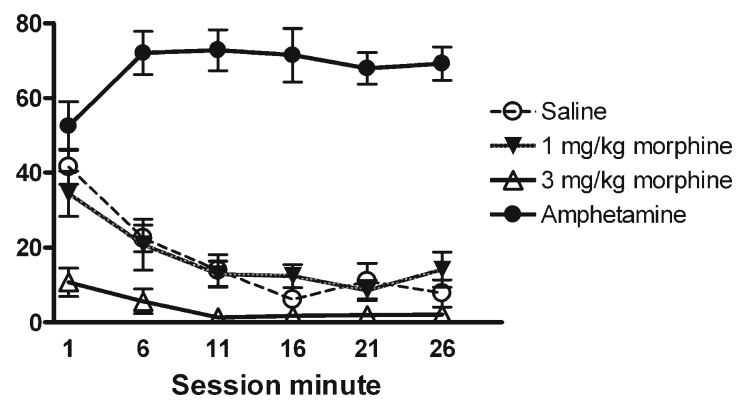

\section{Experiment 3}

USV call rate was markedly inhibited by morphine $(1 \mathrm{mg} /$ $\mathrm{kg}$ ) during the first two conditioning trials (saline vs. morphine on trials 1 and 2: Wilcoxon $Z=2.71$ and 2.63, both $p<$ 0.05; Fig. 7a). Locomotor activity, in contrast, was significantly increased from the second morphine conditioning trial onwards (paired $t$ tests between saline and morphine locomotion on trials 2,3 , and $4: t_{15}=2.65,4.03,3.12$, respectively, $p<0.05-0.01$; Fig. $7 b$ ). On the CPP test day, the subjects greatly preferred the morphine-paired floor texture (one sample $t$ test for MOR-SAL difference score: $t_{15}=5.15, p=0.0001, n=16$; Fig. $8 \mathrm{a}$ ), but they did not call at a greater rate (i.e., calls per min) when located on the drugassociated flooring (Fig. 8b). Finally, AMPH produced a significant increase in calling, as expected (Wilcoxon $Z=$ 2.73, $p<0.01$; Fig. 8c)

\section{Analysis of USV subtypes}

Subtype analyses were performed in all three experiments. The proportion of trill calls was not significantly enhanced by morphine under any experimental condition, and there

Fig. 4 Experiment 1.3 morphine (1 and $3 \mathrm{mg} / \mathrm{kg}, \mathrm{SC})$ significantly increased locomotor activity (a), while dose-dependently decreasing emission of $50-\mathrm{kHz}$ calls (b). Each rat was tested under all conditions $(n=24), * * p<0.01$, $* * * p<0.001$ vs. saline condition a.

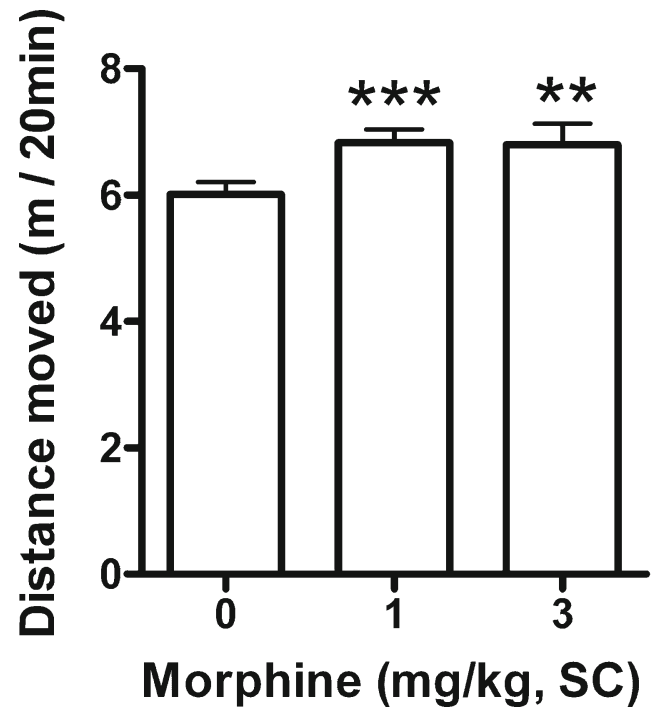

was also no discernible trend in this direction (Supplementary Tables S2, S4, S6, S9, S10, and S11). Similarly, there were no consistent changes in either the absolute number or proportion of the other $50-\mathrm{kHz}$ call subtypes (Supplementary Tables S1-S11); however, overall call rates were quite low, producing a high degree of variability in the proportional measures.

\section{Discussion}

The present study yielded several novel findings. First, morphine failed to increase the $50-\mathrm{kHz}$ call rate under a variety of experimental conditions, i.e., after morphine preexposure, with repeated drug testing, or in different social contexts. Second, low doses of morphine that failed to increase $50-\mathrm{kHz}$ call rates were nevertheless rewarding and non-sedative. Third, detailed call subtype analysis indicated that, contrary to our hypothesis, morphine did not preferentially promote trill calls.

The USV-suppressant effect of morphine, which generalized to pair-tested subjects, did not appear to reflect general behavioral inhibition since it occurred at sub-cataleptic b.

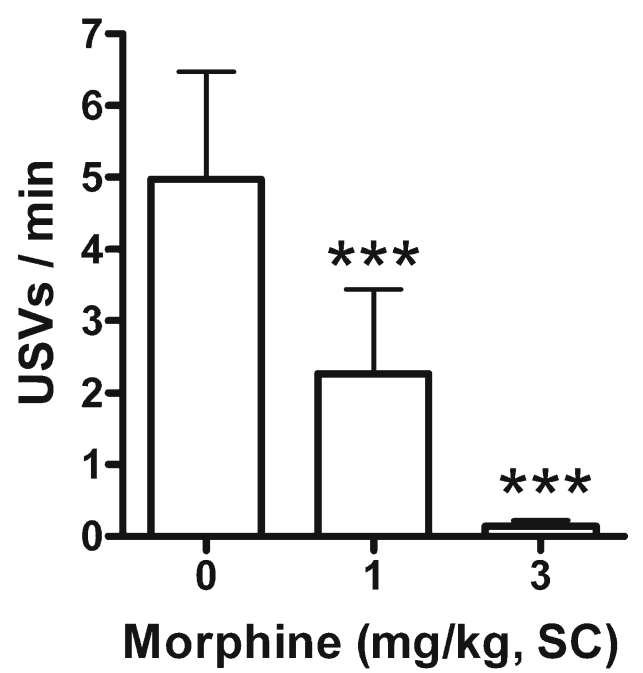


Fig. 5 Experiment 2: call rate was unaffected during morphine conditioning. Each rat received saline (open bars) and morphine, given at $1 \mathrm{mg} / \mathrm{kg}$ $(\mathbf{a}, \mathbf{b})(n=12$, light gray bars $)$ or $3 \mathrm{mg} / \mathrm{kg}(\mathbf{c}, \mathbf{d})(n=12$, dark gray bars). Locomotor activity during conditioning with 1 $\mathrm{mg} / \mathrm{kg}$ (b) and $3 \mathrm{mg} / \mathrm{kg}$ (d) morphine was unaltered during the four conditioning trials, except for an apparent stimulatory effect at the higher dose $(3 \mathrm{mg} / \mathrm{kg})$ on the third and fourth conditioning trial, ${ }^{\wedge} p<0.06$ a.

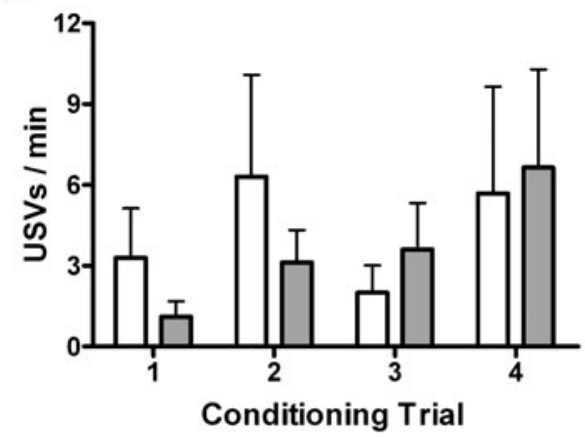

c.

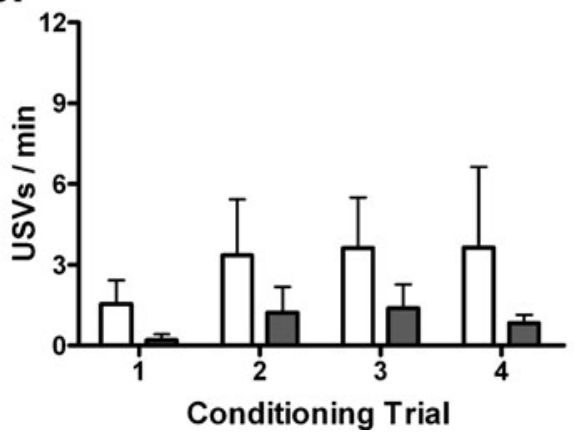

b.

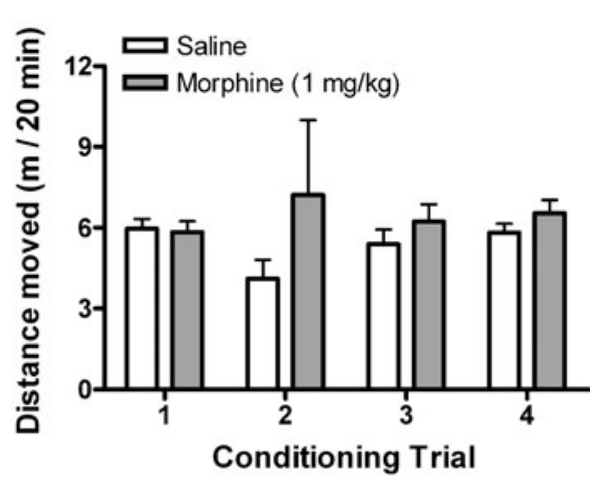

d.

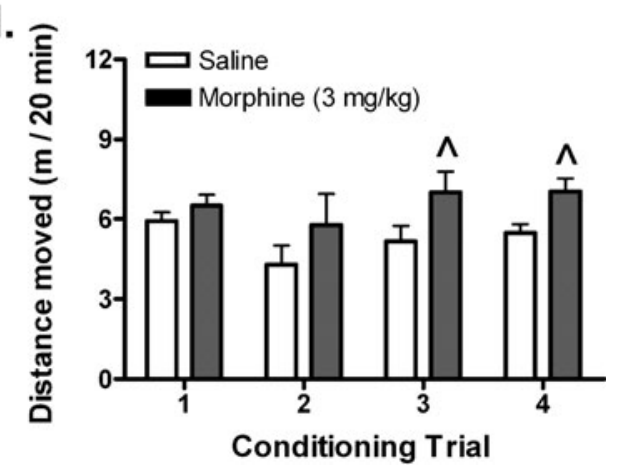

doses (Fernandes et al. 1977; Fog 1970) and was associated with locomotor stimulation rather than sedation (experiments 1.3 and 3). This USV-suppressant effect was unaffected by prior administration of morphine in the home cage (experiment 1.1), whereas it waned rapidly when the drug was later tested repeatedly in the same environment (experiment 1.1 and experiment 3); tolerance to this USV-inhibitory effect was therefore likely context-specific (Siegel and MacRae 1984). The observed tolerance appeared reversible upon drug withdrawal, as evidenced by an inhibition of USVs with morphine after 8 days of abstinence (1.2 vs. 1.3).

Horizontal locomotor activity is inhibited or stimulated by acute systemic administration of morphine, depending on dose and time after injection, with a shift towards locomotor stimulation upon repeated drug testing (Babbini and Davis 1972; Nakamura et al. 1978; Shoaib et al. 1994; Vasko and Domino 1978). Consistent with this literature, a locomotor stimulant effect was immediately apparent in subjects that had already received seven to ten morphine injections (experiment 1.3) whereas such an effect emerged gradually in subjects that were initially drug naïve (experiment 2 and 3 ).

The conditioned place preference paradigm is widely used to study the rewarding properties of drugs and other stimuli (Tzschentke 1998, 2007). In the present study, the two morphine doses ( 1 and $3 \mathrm{mg} / \mathrm{kg}$ ) produced a CPP of similar magnitude, consistent with several published reports (Bardo et al. 1995). Hence, morphine exerted rewarding effects during conditioning but at the same time failed to
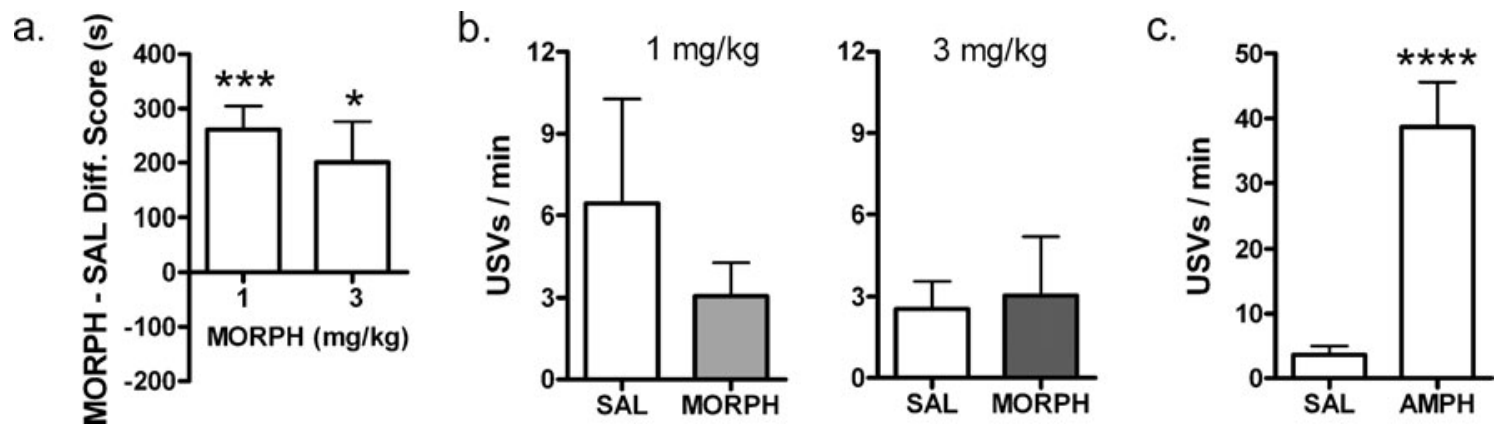

Fig. 6 Experiment 2: post-conditioning and AMPH tests. a On the CPP test day (day 10), both doses of morphine produced a significant place preference. b On days 11 and 12, there was no significant difference in USV emission when rats were confined and when drugfree to the morphine-paired vs. saline-paired floor textures (gray and

open bars, respectively). The $y$-axes represent the mean+SEM USVs per minute, $n=12$ rats per dose. c AMPH markedly increased the call rate in a subsequent test, as expected $(n=24),{ }^{*} p<0.05,{ }^{* * *} p<0.001$, $* * * * p<0.0001$ 
Fig. 7 Experiment 3: during conditioning trials, morphine initially decreased the call rate (a). However, morphine significantly increased locomotor activity during the last three trials (b). $* p<0.05$, $* * p<0.01(n=16)$

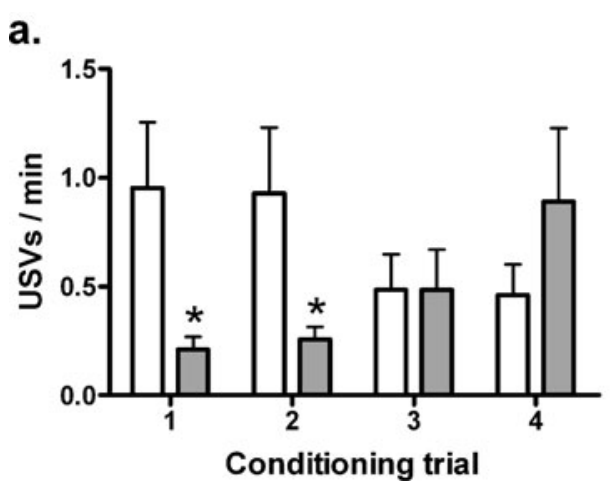

increase USV production. While $50-\mathrm{kHz}$ USVs are associated with a variety of natural and nonnatural appetitive stimuli, it appears that only certain drugs of abuse, namely AMPH, cocaine, and methylphenidate, lead to an increase in USV emission (Ahrens et al. 2009; Maier et al. 2010; Meyer et al. 2011; Simola et al. 2012; Williams and Undieh 2010; Wright et al. 2010, 2012). Thus, neither morphine (Hamed et al. 2012; Simola et al. 2012; present study) nor MDMA (Sadananda et al. 2012) has been found to stimulate adult rat $50-\mathrm{kHz}$ calling after acute administration.

The observed dissociation between morphine-induced CPP and $50-\mathrm{kHz}$ calling indicates that these two behavioral measures are not generally interchangeable. We cannot at present exclude the possibility that, despite considerable correlative evidence (Knutson et al. 2002), 50-kHz calls are unrelated to reward per se. Alternatively, the concept of reward is multi-faceted (Berridge and Robinson 2003) and ultrasonic vocalizations and CPP possibly reflect different aspects of this phenomenon. A third possibility is that $50-\mathrm{kHz}$ calling and CPP normally track the same type of reward, but that this relationship can be obscured by additional drug effect(s). In this regard, we are not aware of any reports that morphine can directly affect vocal cords or respiratory muscles. However, morphine can produce an aversive effect in rats through activation of peripheral opiate receptors (Bechara et al. 1987; Bechara and van der Kooy 1985); conceivably $50-\mathrm{kHz}$ calling, like conditioned taste aversion, is preferentially sensitive to aversive drug effects. Morphine can also depress the activity of locus coeruleus noradrenergic neurons (Bird and Kuhar 1977; Korf et al. 1974); this action may also be pertinent since $50-\mathrm{kHz}$ USV emission is critically dependent on CNS noradrenergic transmission (Wright et al. 2012).

Although morphine administration produced a robust $\mathrm{CPP}$, it failed to produce conditioned ultrasonic calling when the rat was exposed to the drug-paired context, either though passive confinement (experiment 2) or during free choice (experiment 3 ). In experiment 2, the tests of conditioned USVs occurred after the CPP test session, and so it is possible that the reward-cue associations had already extinguished. However, this is unlikely for two reasons. First, CPP is reported to take several drug-free sessions to extinguish after a similar morphine conditioning regime (Mueller et al. 2002; Parker and Mcdonald 2000; Rutten et al. 2011). Second, extinction of morphine CPP is typically contextdependent (Parker et al. 2006), and the USV tests were performed in a separate room and in a distinct apparatus from the CPP test.

The non-occurrence of morphine-conditioned $50-\mathrm{kHz}$ USVs in experiments 2 and 3 stands in contrast to two positive reports of conditioned calls occurring either during the expression of morphine CPP (Knutson et al. 1999) or in rats passively exposed to drug-paired CPP cues (Burgdorf et al. 2001). Several methodological factors could potentially
Fig. 8 Experiment 3: a On the CPP test day, morphine produced a significant place preference. b There was no significant difference in USV emission when rats were on the morphine- vs. saline-paired floor textures during the CPP test (open and gray bars, respectively, mean+SEM USVs per minute). c Tested subsequently, AMPH markedly increased the call rate, as expected. ${ }^{* *} p<0.01$, $* * * * p=0.0001 \quad(n=16)$ a.

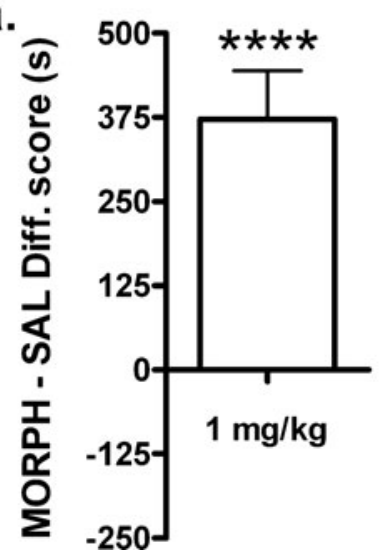

b.

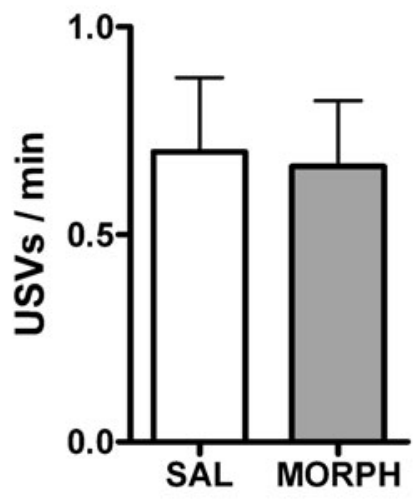

C.

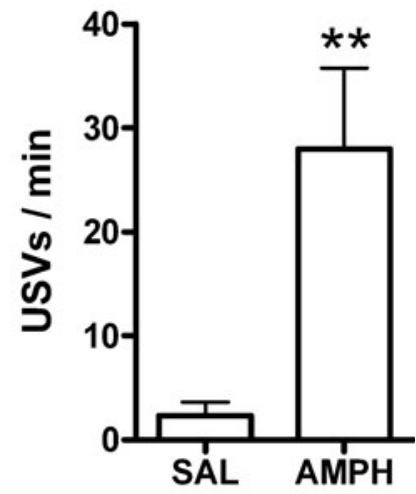


help to explain these disparate findings, for example: the dose of morphine (i.e., $5 \mathrm{mg} / \mathrm{kg}$ ) (Burgdorf et al. 2001), use of visual as well as tactile cues (Burgdorf et al. 2001; Knutson et al. 1999), biased CPP procedure (Knutson et al. 1999), conditioning session duration and number (Knutson et al. 1999), and rat strain (Burgdorf et al. 2001). Perhaps most significantly, however, call counting in these earlier reports was based on heterodyne and/or frequency-divided acoustic signals, which is less reliable than spectrographic analysis of broadband signals (Hamdani and White 2011; Parsons 2000).

\section{Limitations}

Low rates of 50-kHz calling under morphine and saline impeded call subtype analysis. Low call rates, together with high intersubject variability, likely explains why morphine clearly suppressed calling in experiments 1 and 3 , but not in experiment 2 . In this context, it would therefore be interesting to examine the effects of morphine on high rates of $50-\mathrm{kHz}$ USVs elicited by positive stimuli, such as sex-relevant odors (e.g., Ciucci et al. 2007) or AMPH (e.g., Wright et al. 2010).

The doses of morphine used in the present study were chosen because they are reliably rewarding and subcataleptic. We cannot of course exclude the possibility that doses outside the present range, or more extended dosing (Hamed et al. 2012), would produce unconditioned or conditioned USVs. Finally, whether the morphine-induced suppression of USVs is reversible using an opiate antagonist remains to be investigated.

\section{Conclusion}

The findings of the present study clearly show that even if 50$\mathrm{kHz}$ calls, or trill calls in particular, reflect hedonia in some contexts (see "Introduction"), this is not the case after acute morphine administration. Furthermore, while $50-\mathrm{kHz}$ calls are associated with a variety of natural and artificial appetitive stimuli, they also occur in aversive contexts such as aggression (e.g., Haney and Miczek 1994; Vivian and Miczek 1993), morphine withdrawal (Vivian and Miczek 1991), $\mathrm{CO}_{2}$ exposure (Niel and Weary 2006) and pain (Dinh et al. 1999). The present findings provide an additional reason to exert caution when appraising the significance of $50-\mathrm{kHz}$ calls.

Acknowledgments This study was supported by a Natural Science and Engineering Research Council of Canada (NSERC) discovery grant (155055, to P.B.S.C), and an NSERC Postgraduate Scholarship D (to J.M.W). The authors thank Tina Scardochio for constructive comments on the manuscript. P.B.S.C. is a member of the Center for studies in Behavioral Neurobiology at Concordia University in Montreal. The authors have no financial relationship with the organizations that sponsored this research. All experiments comply with the current laws of Canada.

\section{References}

Ahrens AM, Ma ST, Maier EY, Duvauchelle CL, Schallert T (2009) Repeated intravenous amphetamine exposure: rapid and persistent sensitization of $50-\mathrm{kHz}$ ultrasonic trill calls in rats. Behav Brain Res 197:205-209

Angrist B, Sathananthan G, Wilk S, Gershon S (1974) Amphetamine psychosis: behavioral and biochemical aspects. J Psychiatr Res 11:13-23

Babbini M, Davis WM (1972) Time-dose relationships for locomotor activity effects of morphine after acute or repeated treatment. $\mathrm{Br} \mathrm{J}$ Pharmacol 46:213-224

Bardo MT, Rowlett JK, Harris MJ (1995) Conditioned place preference using opiate and stimulant drugs: a meta-analysis. Neurosci Biobehav Rev 19:39-51

Bechara A, van der Kooy D (1985) Opposite motivational effects of endogenous opioids in brain and periphery. Nature 314:533-534

Bechara A, Zito KA, van der Kooy D (1987) Peripheral receptors mediate the aversive conditioning effects of morphine in the rat. Pharmacol Biochem Behav 28:219-225

Berridge KC, Robinson TE (2003) Parsing reward. Trends Neurosci 26:507-513

Biala G, Kruk M (2007) Amphetamine-induced anxiety-related behavior in animal models. Pharmacol Rep 59:636-644

Bird SJ, Kuhar MJ (1977) Iontophoretic application of opiates to the locus coeruleus. Brain Res 122:523-533

Brudzynski SM (2007) Ultrasonic calls of rats as indicator variables of negative or positive states: acetylcholine-dopamine interaction and acoustic coding. Behav Brain Res 182:261-273

Brudzynski SM (2009) Communication of adult rats by ultrasonic vocalization: biological, sociobiological, and neuroscience approaches. ILAR J 50:43-50

Brudzynski SM, Pniak A (2002) Social contacts and production of 50$\mathrm{kHz}$ short ultrasonic calls in adult rats. J Comp Psychol 116:7382

Burgdorf J, Moskal JR (2009) Frequency modulated $50 \mathrm{kHz}$ ultrasonic vocalizations reflect a positive emotional state in the rat: neural substrates and therapeutic implications. In: Brudzynski SM (ed) Handbook of mammalian vocalization. Academic, Oxford, pp 209-214

Burgdorf J, Knutson B, Panksepp J, Shippenberg TS (2001) Evaluation of rat ultrasonic vocalizations as predictors of the conditioned aversive effects of drugs. Psychopharmacology (Berl) 155:35-42

Burgdorf J, Kroes RA, Moskal JR, Pfaus JG, Brudzynski SM, Panksepp J (2008) Ultrasonic vocalizations of rats (Rattus norvegicus) during mating, play, and aggression: behavioral concomitants, relationship to reward, and self-administration of playback. J Comp Psychol 122:357-367

Burgdorf J, Panksepp J, Moskal JR (2010) Frequency-modulated $50 \mathrm{kHz}$ ultrasonic vocalizations: a tool for uncovering the molecular substrates of positive affect. Neurosci Biobehav Rev 35:1831-1836

Calcagnetti DJ, Schechter MD (1992) Place conditioning reveals the rewarding aspect of social interaction in juvenile rats. Physiol Behav 51:667-672

Ciucci MR, Ma ST, Fox C, Kane JR, Ramig LO, Schallert T (2007) Qualitative changes in ultrasonic vocalization in rats after unilateral dopamine depletion or haloperidol: a preliminary study. Behav Brain Res 182:284-289

Covington HEI, Miczek KA (2003) Vocalizations during withdrawal from opiates and cocaine: possible expressions of affective distress. Eur J Pharmacol 467:1-13

Dinh HK, Larkin A, Gatlin L, Piepmeier E Jr (1999) Rat ultrasound model for measuring pain resulting from intramuscularly injected antimicrobials. PDA J Pharm Sci Technol 53:40-43 
Fernandes M, Kluwe S, Coper H (1977) The development of tolerance to morphine in the rat. Psychopharmacology (Berl) 54:197-201

File SE, Hyde JR (1979) A test of anxiety that distinguishes between the actions of benzodiazepines and those of other minor tranquilisers and of stimulants. Pharmacol Biochem Behav 11:65-69

Fog R (1970) Behavioural effects in rats of morphine and amphetamine and of a combination of the two drugs. Psychopharmacologia 16:305-312

Foltin RW, Fischman MW (1991) Assessment of abuse liability of stimulant drugs in humans: a methodological survey. Drug Alcohol Depend 28:3-48

Hamdani S, White NM (2011) Ultrasonic vocalization ratios reflect the influence of motivational state and amygdala lesions on different types of taste avoidance learning. Behav Brain Res 217:88-98

Hamed A, Taracha E, Szyndler J, Krzascik P, Lehner M, Maciejak P, Skorzewska A, Plaznik A (2012) The effects of morphine and morphine conditioned context on $50 \mathrm{kHz}$ ultrasonic vocalisation in rats. Behav Brain Res 229:447-450

Haney M, Miczek KA (1994) Ultrasounds emitted by female rats during agonistic interactions: effects of morphine and naltrexone. Psychopharmacology (Berl) 114:441-448

Haney M, Miczek KA (1995) Delta opioid receptors: reflexive, defensive and vocal affective responses in female rats. Psychopharmacology (Berl) 121:204-212

Jasinski DR, Preston KL (1986) Evaluation of mixtures of morphine and d-amphetamine for subjective and physiological effects. Drug Alcohol Depend 17:1-13

Kaltwasser MT (1990) Acoustic signaling in the black rat (Rattus rattus). J Comp Psychol 104:227-232

Knutson B, Burgdorf J, Panksepp J (1999) High-frequency ultrasonic vocalizations index conditioned pharmacological reward in rats. Physiol Behav 66:639-643

Knutson B, Burgdorf J, Panksepp J (2002) Ultrasonic vocalizations as indices of affective states in rats. Psychol Bull 128:961-977

Korf J, Bunney BS, Aghajanian GK (1974) Noradrenergic neurons: morphine inhibition of spontaneous activity. Eur J Pharmacol 25:165-169

Lett BT (1989) Repeated exposures intensify rather than diminish the rewarding effects of amphetamine, morphine, and cocaine. Psychopharmacology (Berl) 98:357-362

Litvin Y, Blanchard DC, Blanchard RJ (2007) Rat $22 \mathrm{kHz}$ ultrasonic vocalizations as alarm cries. Behav Brain Res 182:166-172

Maier EY, Ahrens AM, Ma ST, Schallert T, Duvauchelle CL (2010) Cocaine deprivation effect: cue abstinence over weekends boosts anticipatory $50-\mathrm{kHz}$ ultrasonic vocalizations in rats. Behav Brain Res 214:75-79

Meyer PJ, Ma ST, Robinson TE (2011) A cocaine cue is more preferred and evokes more frequency-modulated $50-\mathrm{kHz}$ ultrasonic vocalizations in rats prone to attribute incentive salience to a food cue. Psychopharmacology (Berl) 219:999-1009

Mueller D, Perdikaris D, Stewart J (2002) Persistence and druginduced reinstatement of a morphine-induced conditioned place preference. Behav Brain Res 136:389-397

Nakamura H, Ishii K, Shimizu M (1978) Some altered responses in rats formerly dependent on morphine. Psychopharmacology (Berl) 56:269-277

Niel L, Weary DM (2006) Behavioural responses of rats to gradual-fill carbon dioxide euthanasia and reduced oxygen concentrations. Appl Anim Behav Sci 100:295-308

Panksepp J, Burgdorf J (2000) 50-kHz chirping (laughter?) in response to conditioned and unconditioned tickle-induced reward in rats: effects of social housing and genetic variables. Behav Brain Res 115:25-38

Parker LA, Mcdonald RV (2000) Reinstatement of both a conditioned place preference and a conditioned place aversion with drug primes. Pharmacol Biochem Behav 66:559-561
Parker LA, Limebeer CL, Slomke J (2006) Renewal effect: contextdependent extinction of a cocaine- and a morphine-induced conditioned floor preference. Psychopharmacology (Berl) 187:133137

Parsons S (2000) Advantages and disadvantages of techniques for transforming and analyzing chiropteran echolocation calls. J Mammal 81:927-938

Pellow S, Chopin P, File SE, Briley M (1985) Validation of open: closed arm entries in an elevated plus-maze as a measure of anxiety in the rat. J Neurosci Meth 14:149-167

Robinson TE, Becker JB (1986) Enduring changes in brain and behavior produced by chronic amphetamine administration: a review and evaluation of animal models of amphetamine psychosis. Brain Res 396:157-198

Rutten K, van der Kam EL, De VJ, Tzschentke TM (2011) Critical evaluation of the use of extinction paradigms for the assessment of opioid-induced conditioned place preference in rats. Pharmacology 87:286-296

Sadananda M, Natusch C, Karrenbauer B, Schwarting RK (2012) 50 $\mathrm{kHz}$ calls in rats: effects of MDMA and the 5-HT(1A) receptor agonist 8-OH-DPAT. Pharmacol Biochem Behav 101:258-264

Sales GD, Pye D (1974) Ultrasonic communication by animals. Chapman and Hall, London

Shoaib M, Stolerman IP, Kumar RC (1994) Nicotine-induced place preferences following prior nicotine exposure in rats. Psychopharmacology (Berl) 113:445-452

Siegel S, MacRae J (1984) Environmental specificity of tolerance. Trends Neurosci 7:140-143

Simola N, Ma ST, Schallert T (2009) Influence of acute caffeine on 50$\mathrm{kHz}$ ultrasonic vocalizations in male adult rats and relevance to caffeine-mediated psychopharmacological effects. Int J Neuropsychopharmacol 13:123-132

Simola N, Fenu S, Costa G, Pinna A, Plumitallo A, Morelli M (2012) Pharmacological characterization of $50-\mathrm{kHz}$ ultrasonic vocalizations in rats: comparison of the effects of different psychoactive drugs and relevance in drug-induced reward. Neuropharmacology 63:224-234

Tzschentke TM (1998) Measuring reward with the conditioned place preference paradigm: a comprehensive review of drug effects, recent progress and new issues. Prog Neurobiol $56: 613-672$

Tzschentke TM (2007) Measuring reward with the conditioned place preference (CPP) paradigm: update of the last decade. Addict Biol $12: 227-462$

Vasko MR, Domino EF (1978) Tolerance development to the biphasic effects of morphine on locomotor activity and brain acetylcholine in the rat. J Pharmacol Exp Ther 207:848-858

Vivian JA, Miczek KA (1991) Ultrasounds during morphine withdrawal in rats. Psychopharmacology (Berl) 104:187-193

Vivian JA, Miczek KA (1993) Morphine attenuates ultrasonic vocalization during agonistic encounters in adult male rats. Psychopharmacology (Berl) 111:367-375

Williams SN, Undieh AS (2010) Brain-derived neurotrophic factor signaling modulates cocaine induction of rewardassociated ultrasonic vocalization in rats. J Pharmacol Exp Ther 332:463-468

Wohr M, Schwarting RK (2009) Ultrasonic communication in rats: effects of morphine and naloxone on vocal and behavioral responses to playback of $50-\mathrm{kHz}$ vocalizations. Pharmacol Biochem Behav 94:285-295

Wohr M, Schwarting RK (2010) Rodent ultrasonic communication and its relevance for models of neuropsychiatric disorders. eNeuroforum 1:71-80

Wohr M, Houx B, Schwarting RK, Spruijt B (2008) Effects of experience and context on $50-\mathrm{kHz}$ vocalizations in rats. Physiol Behav 93:766-776 
Wright JM, Gourdon JC, Clarke PB (2010) Identification of multiple call categories within the rich repertoire of adult rat $50-\mathrm{kHz}$ ultrasonic vocalizations: effects of amphetamine and social context. Psychopharmacology (Berl) 211:1-13

Wright JM, Dobosiewicz MR, Clarke PB (2012) Alpha- and betaadrenergic receptors differentially modulate the emission of spontaneous and amphetamine-induced $50-\mathrm{kHz}$ ultrasonic vocalizations in adult rats. Neuropsychopharmacology 37:808-821

Zacny JP, Lichtor JL, Flemming D, Coalson DW, Thompson WK (1994) A dose-response analysis of the subjective, psychomotor and physiological effects of intravenous morphine in healthy volunteers. J Pharmacol Exp Ther 268:1-9 